\title{
Compound anisodine affects the proliferation and calcium overload of hypoxia-induced rat retinal progenitor cells and brain neural stem cells via the $\mathrm{p}-\mathrm{ERK} 1 / 2 / \mathrm{HIF}-1 \alpha / \mathrm{VEGF}$ pathway
}

\author{
QUN WANG $^{1}$, SHAN GAO ${ }^{1}$, YU LUO $^{2}$ and QIAN-YAN KANG ${ }^{1}$ \\ ${ }^{1}$ Department of Ophthalmology, The First Affiliated Hospital, Medical School of Xi'an Jiaotong University; \\ ${ }^{2}$ Environment and Genes Related to Diseases Key Laboratory of Education Ministry, College of \\ Medicine, Xi'an Jiaotong University, Xi'an, Shaanxi 710061, P.R. China
}

Received January 7, 2016; Accepted January 6, 2017

DOI: $10.3892 /$ etm.2017.4528

\begin{abstract}
As a Traditional Chinese Medicine, compound anisodine (CA) has previously been shown to regulate the vegetative nervous system, improve microcirculation and scavenge reactive oxygen species, and has been commonly utilized as a neuroprotective agent to treat ischemic optic neuropathy and choroidoretinopathy. The present study aimed to investigate the neuroprotective effects of CA on the proliferation and calcium overload of hypoxia-induced rat retinal progenitor cells (RPCs) and brain neural stem cells (BNSCs) harvested from neonatal Sprague-Dawley rats. Cells were treated with CA at 0.126, 0.252, 0.505 or $1.010 \mathrm{~g} / \mathrm{l}$ for four hours prior to or after hypoxia $(<1 \%$ oxygen) for four h, followed by re-oxygenation for four hours; a normal control group and a CA-untreated hypoxia model group were also included. An MTT assay demonstrated that the cell viability was markedly improved following treatment with 0.126-1.010 g/l CA, compared with that in the hypoxia model group $(\mathrm{P}<0.05)$. Bromodeoxyuridine $(\mathrm{BrdU})$ immunocytochemical staining and flow cytometry indicated that after culture in hypoxia for $4 \mathrm{~h}$, the number of $\mathrm{BrdU}^{+} \mathrm{RPCs}$ and BNSCs was significant decreased, as well as the cell population in $\mathrm{S}+\mathrm{G} 2$ phase of the cell cycle, which was significantly attenuated by treatment with $1.010 \mathrm{~g} / \mathrm{l} \mathrm{CA}$ for $4 \mathrm{~h}$ prior to hypoxia $(\mathrm{P}<0.05)$. Furthermore, laser scanning confocal microscopy showed that the intracellular calcium concentration in hypoxia-cultured RPCs and BNSCs was markedly increased, which was attenuated by $0.126-1.010 \mathrm{~g} / \mathrm{l} \mathrm{CA}$ in a concentration-dependent manner $(\mathrm{P}<0.05)$. Furthermore, western blot analysis demonstrated that after hypoxia, the protein levels of hypoxia-inducible factor (HIF)-1 $\alpha$ and vascular endothelial growth factor (VEGF) were
\end{abstract}

Correspondence to: Dr Qian-Yan Kang, Department of Ophthalmology, The First Affiliated Hospital, Medical School of Xi'an Jiaotong University, 28 Xianning West Road, Xi'an, Shaanxi 710061, P.R. China

E-mail: kangqy@mail.xjtu.edu.cn

Key words: retinal progenitor cells, brain neural stem cells, hypoxia, calcium overload, compound anisodine upregulated in RPCs and BNSCs, whereas phosphorylated extracellular signal-regulated kinase (phospho-ERK 1/2 and Cyclin D1 were downregulated; of note, treatment with $1.010 \mathrm{~g} / 1 \mathrm{CA}$ significantly attenuated these changes $(\mathrm{P}<0.05)$. The results of the present study suggested that CA may improve the proliferation and inhibit calcium overload in hypoxia-induced RPCs and BNSCs by altering the protein levels of Cyclin D1 as well as signaling through the $\mathrm{p}-\mathrm{ERK} 1 / 2 / \mathrm{HIF}-1 \alpha / \mathrm{VEGF}$ pathway.

\section{Introduction}

Numerous ocular diseases, including age-associated macular degeneration, glaucoma and retinitis pigmentosa, cause a severe and irreversible loss of visual function. Patients with these conditions suffer progressive visual decline resulting from irreversible loss of retinal neurons; however, at present, no therapies are available to repair or replace the damaged retinal cells (1). Advances in molecular biology have identified innovative approaches, including stem cell therapy, which may potentially repair or regenerate diseased retina and subsequently restore visual function in eyes with degenerative retinal disorders $(2,3)$. Various cell sources for replacement of retinal neurons have been identified, including retinal progenitor cells (RPCs), brain neural stem cells (BNSCs), embryonic stem cells, induced pluripotent stem cells and mesenchymal stem cells $(4,5)$. Among these, RPCs and BNSCs, which are derived from the committed central nervous tissue, are two promising types of stem cell for retinal replacement therapy. These cells can be expanded to generate large numbers of cells, which can then be differentiated into major neural retinal cell types, including photoreceptor cells $(6,7)$. When transplanted, RPCs and BNSCs are incorporated into the neural retina have been shown to rescue vision in animal models. Furthermore, it was revealed that RPCs from younger donors show better integration than those derived from older donors, and more extensive integration occurs when the host retina is diseased or injured $(8,9)$. As with RPCs, integration of BNSCs is increased when transplanted into young or injured host retina; however, BNSCs transplanted into healthy adult monkeys showed little migration or integration, forming a monolayer of stable BNSCs $(10,11)$. This phenomenon suggested that more attention 
should be paid to changes in the local micro-environment that occur in response to degeneration, trauma or ischemia, hypoxia and ischemia/reperfusion, which lead to pathological changes that produce free radicals, intracellular calcium overload and finally induce cell apoptosis.

Compound anisodine (CA) is a Traditional Chinese Medicine, which is a compound preparation made from hydrobromide anisodine and procaine hydrochloride. CA have previously been shown to regulate the vegetative nervous system, improve microcirculation, scavenge reactive oxygen species and has been commonly utilized as a neuroprotective agent to treat primary and secondary chemic optic neuropathy and choroidoretinopathy, including central retinal vein occlusion, occlusion, blepharospasm, glaucoma, optic atrophy, senile macular degeneration, childhood amblyopia, improving the anterior ischemic optic neuropathy of the vessels, reducing vascular resistance and restoring patients' vision (12-15). However, whether CA has an effect on stem cells has remained elusive. The present study aimed to investigate the neuroprotective effects of CA by assessing its restorative effects on the proliferation and calcium overload of hypoxia-induced rat RPCs and BNSCs.

\section{Materials and methods}

Isolation and culture of neonatal rat RPCs and BNSCs. A total of eighty neonatal Sprague Dawley (SD) rats on postnatal day 0 (P0) were obtained from the Laboratory Animal Center of Xi'an Jiaotong University Health Science Center (Xi'an, China) and housed in a room with a constant temperature of $24^{\circ} \mathrm{C}$ and a relative humidity of $50 \pm 15 \%$ under a 12 -h light/dark cycle, and handling protocols were approved by the Institutional Animal Care and Use Committee of Xi'an Jiaotong University Health Science Center. Eyes and brains from 80 neonatal SD rats, which were sacrificed by decapitation were enucleated and washed several times with PBS. The neuroretinas and cerebral cortex were respectively dissected from each eye and the brain, minced into small pieces, centrifuged at $398.3 \mathrm{x} \mathrm{g}$ at $4^{\circ} \mathrm{C}$ for $5 \mathrm{~min}$ and resuspended. Suspensions of RPCs and BNSCs were collected after passing them through a $100-\mathrm{mm}$ mesh strainer, followed by culture in Dulbecco's Modified Eagle's Medium/F12 medium [10 ng/ml recombinant human endothelial growth factor, $20 \mathrm{ng} / \mathrm{ml}$ recombinant human basic fibroblast growth factor, 2\% B27 supplement, 1\% N2 supplement, $100 \mathrm{U} / \mathrm{ml}$ penicillin and $100 \mu \mathrm{g} / \mathrm{ml}$ streptomycin (Gibco; Thermo Fisher Scientific, Inc., Waltham, MA, USA)] at $37^{\circ} \mathrm{C}$ with $5 \% \mathrm{CO}_{2}$ and $90 \%$ humidity. The RPC and BNSC populations were enriched based on their ability to form neurospheres within 3-5 days, and the cultures were passaged every 2-3 days by mechanical trituration to obtain single cell suspensions that were then diluted 1:2 with fresh culture medium. The cells were collected at the first passage and cultured in 96-well plates at a density of $5-10 \times 10^{3}$ cells per well in $0.2 \mathrm{ml}$ medium, in 24-well plates with polylysine-coated glass slides in each well at a density of $5-10 \times 10^{4}$ cells per well in $0.5 \mathrm{ml}$ medium or in 6 -well plates at a density of $5-10 \times 10^{5}$ cells per well in $2 \mathrm{ml}$ medium.

Identification of RPCs and BNSCs by fluorescent immunocytochemistry. The RPCs and BNSCs were characterized by immunofluorescence staining for progenitor and eye field developmental marker paired box (Pax)6 and neural stem cell-specific marker Nestin. Cells were fixed with $4 \%$ paraformaldehyde, washed three times with $1 \mathrm{X}$ PBS, incubated in blocking buffer $(0.3 \%$ Triton $\mathrm{X}-100$ in $5 \%$ goat serum (Invitrogen; Thermo Fisher Scientific, Inc.) for $1 \mathrm{~h}$ at room temperature and stained with primary antibodies [mouse monoclonal to Nestin (cat. no. 2Q178, 1:200 dilution; Abcam, Cambridge, MA, USA) and rabbit polyclonal to Pax6 (cat. no. ab5790; 1:100 dilution, Abcam)] overnight at $4^{\circ} \mathrm{C}$ in blocking buffer. The cells were then washed three times with $1 \mathrm{X}$ PBS, stained with secondary antibodies [goat Cy3-conjugated anti-mouse or goat fluorescein isothiocyanate (FITC)-conjugated anti-rabbit (ZF-0511, Alexa Fluor 488, 1:200 dilution and ZF-0513, Alexa Fluor 594, 1:200 dilution; Zhongshan Goldenbridge Biotechnology, Co., Ltd., Beijing, China),] for one hour at room temperature. Subsequent to counterstaining with DAPI for five min, cells were washed three times with $1 \mathrm{X}$ PBS prior to imaging. The fluorescent images were captured using an inverted fluorescence microscope (DP71; Olympus Corp., Tokyo, Japan).

Grouping. The harvested rat neuronal RPCs and BNSCs were divided into 10 groups as follows: i) NC, normal control group, in which the cells were cultured under normal conditions for $4 \mathrm{~h}$; ii) hypoxia model (HM) group, in which cells were cultured in an incubator at $37^{\circ} \mathrm{C}$, containing $5 \% \mathrm{CO}_{2}+95 \% \mathrm{~N}_{2}(<1 \%$ oxygen) and saturated humidity for $4 \mathrm{~h}$; iii-vi) Pre-hypoxia groups, treated with CA (Beijing Zizhu Pharmaceutical Co., Ltd., Beijing, China) at various concentrations (C1, 0.126; $\mathrm{C} 2,0.252$; C3, 0.505; C4, $1.010 \mathrm{~g} / \mathrm{l})$ prior to hypoxia; vii-x) Post-hypoxia groups, treated with CA (C1-4) after hypoxia. The Pre-hypoxia groups were treated with CA just before the 4 h-hypoxia, and the Post-hypoxia groups were treated with different concentrations of CA at the start of reoxygenation following being cultured in hypoxia for $4 \mathrm{~h}$. Both groups were assessed in the subsequent assays following $4 \mathrm{~h}$ of culture in normal conditions. The morphology images were captured using an inverted phase contrast microscope (CKX41; Olympus Corp.).

Cell viability assay. Cell viability was determined by an MTT assay (Boster Biological Techonology Inc., Wuhan, China) in 96-well plates at $24 \mathrm{~h}$ after the cells were treated. In brief, cells were incubated with $20 \mu \mathrm{l}$ MTT reagent in $100 \mu$ l culture media for $4 \mathrm{~h}$ at $37^{\circ} \mathrm{C}$. After centrifugation at $398.3 \mathrm{x} \mathrm{g}$, the culture media was removed, $0.15 \mathrm{ml}$ dimethylsulfoxide was added to each well and the plates were agitated for $10 \mathrm{~min}$. Optical density (OD) values were measured at $490 \mathrm{~nm}$ using an automatic enzyme-linked immunity analyzer (SS228BEPIII, Beijing Chinese and Western technology Co., Ltd., Beijing, China). The survival rate was calculated as follows: Survival rate $(\%)=\left(\mathrm{OD}_{\text {drug group }}-\mathrm{OD}_{\mathrm{HM} \text { group }}\right) /\left(\mathrm{OD}_{\mathrm{NC} \text { group }}-\mathrm{OD}_{\mathrm{HM} \text { group }}\right) \times 100 \%$.

Bromodeoxyuridine (BrdU) incorporation assay. To assess the proliferation of the treated RPCs and BNSCs, the cultures were incubated with $10 \mu \mathrm{M}$ BrdU (Sigma-Aldrich; Merck KGaG, Darmstadt, Germany; cat. no. B-5002) for $4 \mathrm{~h}$ and then processed for immunofluorescence staining. The cells were fixed in $4 \%$ paraformaldehyde, permeabilized with 
blocking buffer and then treated with $2 \mathrm{~N} \mathrm{HCl}$ in $37^{\circ} \mathrm{C}$ for $40 \mathrm{~min}$, followed by incubation with $0.1 \mathrm{M}$ borate buffer ( $\mathrm{pH}$ 8.5) at room temperature for $12 \mathrm{~min}$. The cells were stained with mouse monoclonal antibody to BrdU (cat. no. Bu20a; 1:1,000 dilution; Cell Signaling Technology, Inc., Danvers, MA, USA) overnight at $4{ }^{\circ} \mathrm{C}$ followed by incubation with goat FITC-conjugated anti-mouse antibody (1:200 dilution; Zhongshang Goldenbridge Biotechnology, Co., Ltd.). In each group, ten fields of view were observed under the microscope for the counting of $\mathrm{BrdU}^{+}$cells.

Cell cycle analysis by flow cytometry (FCM). The cells were grown in 6-well plates at a density of $1 \times 10^{6}$ cells/well. Following incubation at $37^{\circ} \mathrm{C}$ for $24 \mathrm{~h}$, cells were divided into four groups: Normal control group, hypoxia model group, C4 Pre-hypoxia group and $\mathrm{C} 4$ post-hypoxia group. Then, cells were cultured following hypoxia for $48 \mathrm{~h}$ under normoxia condition. RPCs were collected and cell suspensions were fixed using $75 \%$ ethanol. Fixed cells were then treated with $0.1 \mathrm{~g} / 1 \mathrm{RNaseA}$ (Sigma-Aldrich; Merck KGaG) and stained with $0.1 \mathrm{~g} / 1$ propidium iodide (Sigma-Aldrich; Merck KGaG). At least 10,000 events were acquired on a BD FACSCalibur ${ }^{\circledR}$ flow cytometer (BD Biosciences, Franklin Lakes, NJ, USA) with an excitation/emission wavelength of 488/630 nm in order to determine the proportion of cell cycle distribution $[(\mathrm{S}+\mathrm{G} 2) \%]$. The results were analyzed using CellQuest ${ }^{\mathrm{TM}}$ Pro Software (Version 5.1; BD Biosciences).

Measurement of the intracellular calcium concentration $\left(\left[\mathrm{Ca}^{2+}\right]_{i}\right) .\left[\mathrm{Ca}^{2+}\right]_{\mathrm{i}}$ was measured with the membrane-permeant acetoxymethyl ester (AM) form of the $\mathrm{Ca}^{2+}$-sensitive fluorescent dye Fluo 4 (Fluo 4-AM), and the fluorescence intensity was measured via laser scanning confocal microscopy (LSCM). The cultures were loaded with $3 \mu \mathrm{M}$ Fluo 4-AM (Dojindo, Kumamoto, Japan), which was first dissolved in dimethyl sulfoxide with Pluornic F-127 (0.05\%) and incubated for $45 \mathrm{~min}$ at room temperature in D-Hank's solution. Following washing off any extracellular Fluo 4-AM, the samples were incubated in D-Hank's solution for another $25 \mathrm{~min}$. Fluorescence measurement was performed using LSCM (Q550-CW; Leica, Wetzlar, Germany) with excitation/emission wavelengths of $494 / 516 \mathrm{~nm}$. The images were analyzed using the software provided with the LSCM system, with the average fluorescence per unit randomly measured. Each experiment was performed in triplicate and in two independent assays.

Western blot analysis. After treatment with CA for $4 \mathrm{~h}$, cultures were harvested for protein analysis by western blot by lysis in radioimmunoprecipitation assay buffer (Beyotime Institute of Biotechnology, Shanghai, China) and the protein concentration was determined using the bicinchoninic acid protein assay kit (BCA1 AND B9643, Sigma-Aldrich; Merck $\mathrm{KGaG})$. Protein $(80 \mu \mathrm{g})$ was separated by $8 \%$ SDS-PAGE, transferred to a polyvinylidene difluoride membrane (Bio-Rad Laboratories, Inc., Hercules, CA, USA) and blocked with $8 \%$ non-fat milk in Tris-buffered saline containing Tween 20 (TBST) for $1 \mathrm{~h}$ at room temperature. Subsequently, blots were stained with the primary antibodies (rabbit anti-cyclin D1 antibody, 1:1,000, ab134175; mouse anti-HIF-1 $\alpha, 1: 1,000$, ab113642; rabbit anti-VEGF receptor 1, 1:1,000, ab32152; rabbit anti-phospho-ERK 1/2 ${ }^{\text {Thr202/Tyr204 }}, 1: 1,000$, ab214362; rabbit anti-ERK 1/2, 1:2,000, ab196883; mouse anti- $\beta$-actin, 1:20,000, ab8226; Abcam, Cambridge, UK) at $4^{\circ} \mathrm{C}$ overnight. After washing with TBST, the membrane was incubated with horseradish peroxidase-conjugated anti-rabbit immunoglobulin G (1:100,000 dilution; Abcam) at room temperature for $1 \mathrm{~h}$. Resulting bands were imaged with ECL Plus (Merck Millipore KGaA, Darmstadt, Germany) and CL-Xposure film (Thermo Fisher Scientific, Inc.), and band density was measured using Image J software version 1.48 (National Institutes of Health, Bethesda, MD, USA).

Statistical analysis. Values are expressed as the mean \pm standard deviation. One-way analysis of variance and Dunnet's post hoc test were applied to the means in order to determine statistical differences between experimental groups in RPCs or BNSCs. Moreover, a Student's t-test was performed to examine whether there were any differences between RPCs and BNSCs. All statistical analyses were performed using SPSS software, version 17.0 (SPSS, Inc., Chicago, IL, USA). $\mathrm{P}<0.05$ was considered to indicate a statistically significant difference.

\section{Results}

Isolation and characterization of rat RPCs and BNSCs. Rat RPCs and BNSCs were isolated from SD rats at P0, and appeared as mulberry-like neurospheres suspended in the medium with strong refraction. With increasing time in culture, the size of the neurospheres increased; within 3-4 days, the large clumps of spheres became dark in the center, indicating that they were ready to be passaged. If left in culture for 7-8 days at passage 2 , the irregular microspheres adhered to the wells and differentiated into dendritic-like nerve cells. To further characterize the neural linage differentiation capability of rat RPCs and BNSCs, cells were subjected to immunofluorescence staining for progenitor and eye field developmental marker Pax6 and neural stem cell-specific marker Nestin. Immunocytochemistry revealed that RPC and BNSC neurospheres showed high levels of Nestin and Pax6 (Fig. 1).

CA improves the viability of RPCs and BNSCs under hypoxia. After culture in hypoxia ( $<1 \%$ oxygen) for $4 \mathrm{~h}$, the neurospheres aggregated to form large and irregular clusters, which became dark in the center, indicating that an increasing number of cells were dying; at the same time, the amount of floating cell debris visible in the medium increased. The morphological changes of the neurospheres were markedly improved following treatment with 0.126-1.010 g/l CA, compared with the hypoxia model group.

The cultured RPCs and BNSCs were treated with $\mathrm{CA}$ for $4 \mathrm{~h}$ prior to or after hypoxia, after which the cells were cultured under normal oxygen conditions for $24 \mathrm{~h}$. MTT detection demonstrated that following treatment with 0.126-1.010 g/l CA, the cell viability was markedly improved compared with that in the HM group. In the C2, C3 and C4 concentration groups of RPCs and the $\mathrm{C} 3$ and $\mathrm{C} 4$ concentration groups of BNSCs, the cell viability in the pre-hypoxia group was significantly higher as compared with that in the post-hypoxia group. Furthermore, regardless of whether CA 
A
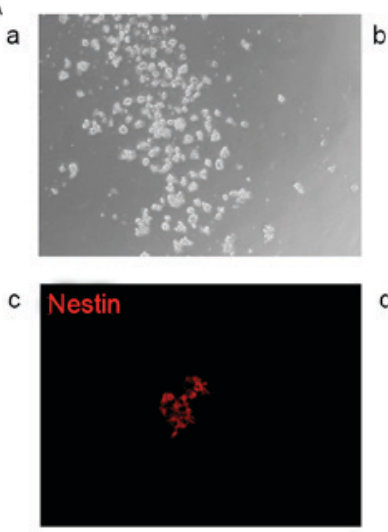

e

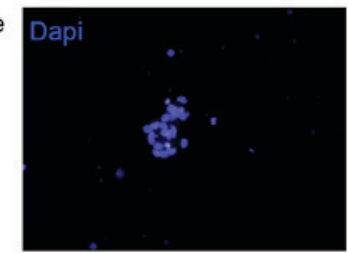

b
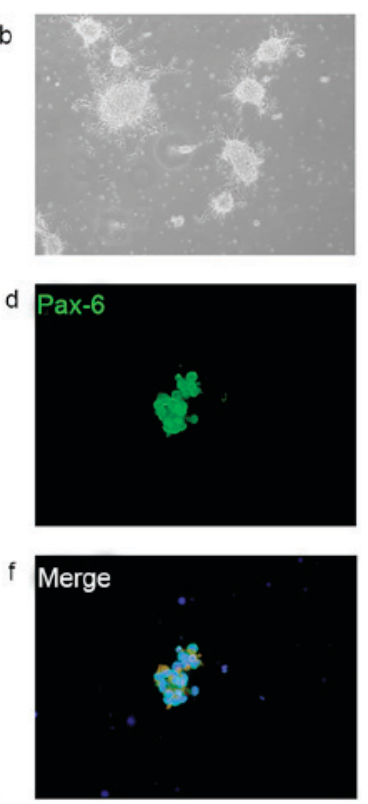

B
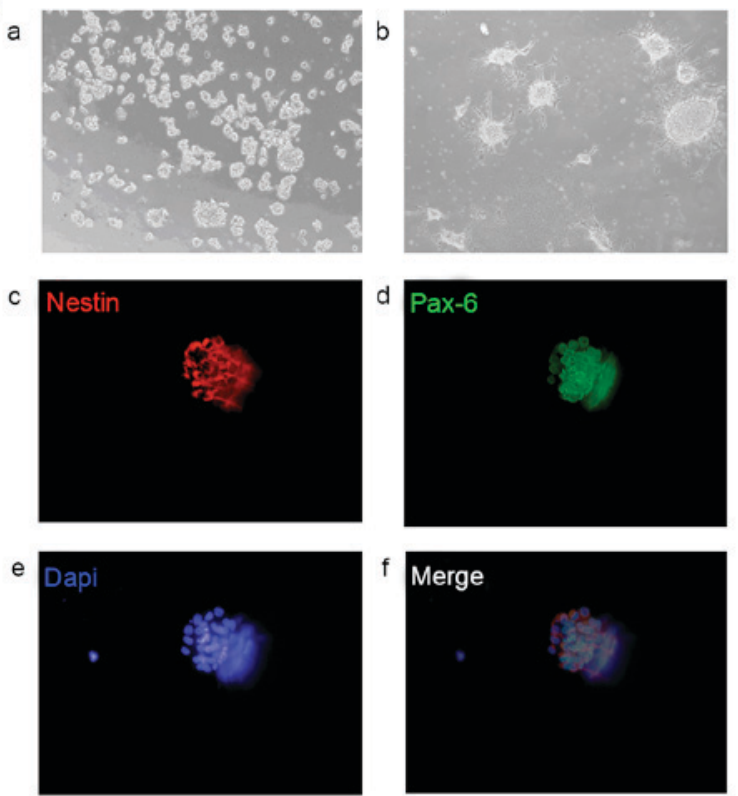
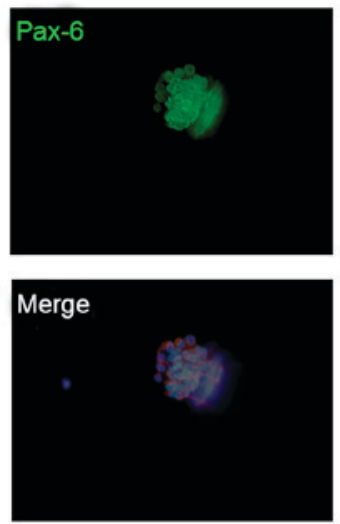

Figure 1. Morphology of rat RPCs and BNSCs, and immunocytochemical expression of Nestin and Pax6. (A) Primary culture of Rat RPCs: (a) Mulberry-like RPC clusters with strong refraction three days after isolation; (b) RPCs cultured for 8 days at passage 2, cells formed irregular microspheres that adhered to the wells and differentiated into dendritic-like nerve cells; (c-f) immunocytochemical detection of the expression of progenitor and eye field developmental marker Pax6 and neural stem cell-specific marker Nestin in primary cultured RPCs at 5 days after isolation. Almost all of the RPCs in the visual field were double positive for (c) nestin (d) and Pax6. (B) Primary culture of Rat BNSCs: (a) The BNSC neurospheres were suspended in the medium, with a larger diameter than that of the RPCs three days after isolation; (b) BNSCs cultured for 8 days at passage 2 also formed irregular neurospheres which adhered to the wells as dendritic-like nerve cells; (c-f) immunocytochemical detection of the expression of Pax6 and nestin in primary cultured BNSCs at 5 days after isolation. Representative phase contrast images are shown (magnification, x200). PRCs, retinal progenitor cells; BNSCs, brain neural stem cells; Pax6, paired box 6.

A

a

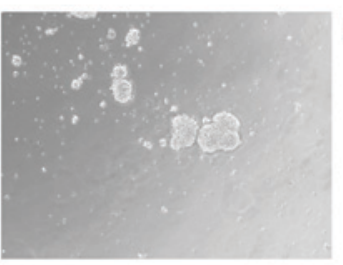

C

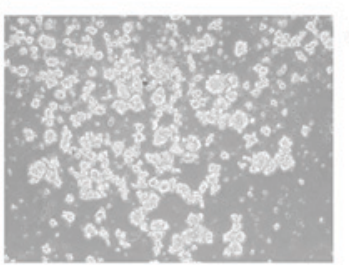

e

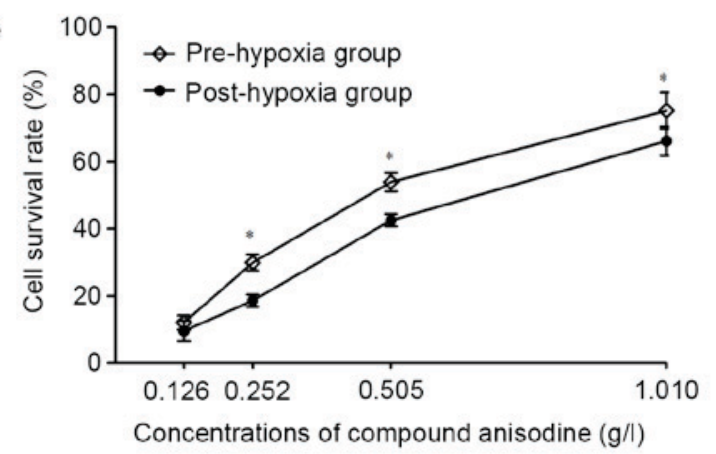

B

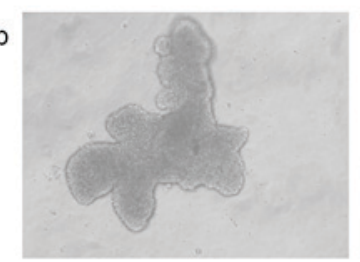

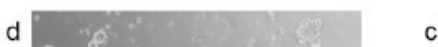
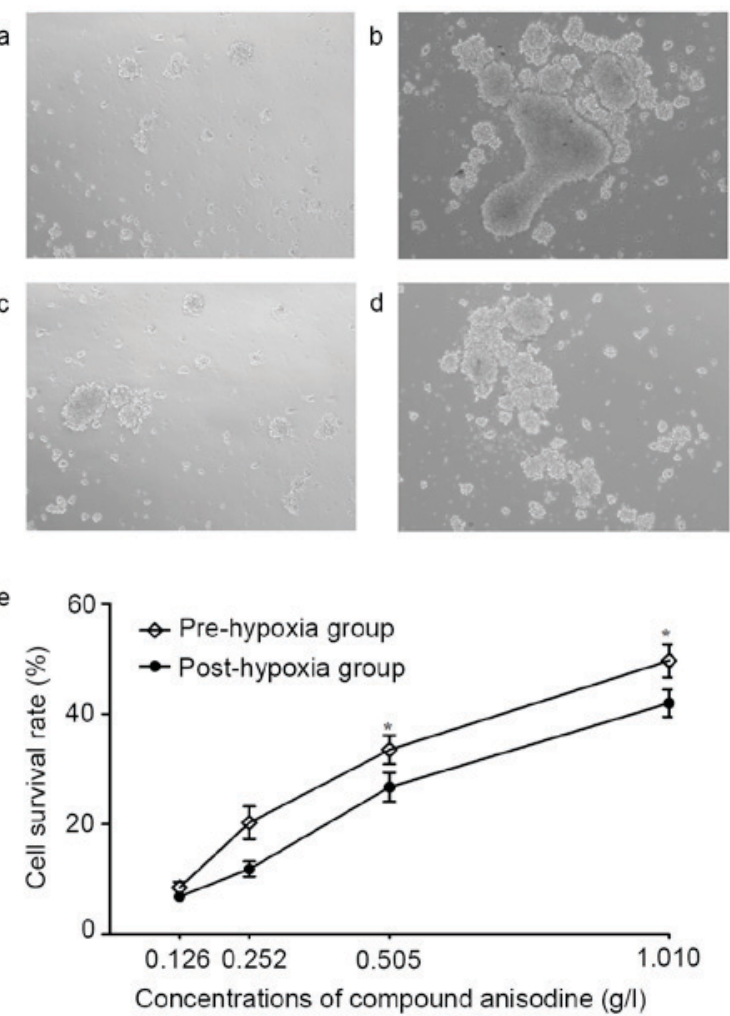

Figure 2. Effects of CA on morphological changes and cell survival rate of hypoxia-cultured RPCs and BNSCs. (A) Effects of CA on the morphological changes and cell survival rate of hypoxia-cultured RPCs; (a) normal control group of RPCs; (b) hypoxia model group of RPCs; (b) C4 pre-hypoxia group of BNSCs; (d) C4 post-hypoxia group of BNSCs; (e) effects of 0.126-1.010 g/l CA on the survival rate of hypoxia-cultured RPCs. (B) Effects of CA on the morphological changes and survival rate of hypoxia-cultured BNSCs; (a) normal control group of BNSCs; (b) hypoxia model group of BNSCs; (b) C4 pre-hypoxia group of BNSCs; (d) C4 post-hypoxia group of BNSCs; (e) effects of 0.126-1.010 g/l CA on cell survival rate of hypoxia-cultured BNSCs. Representative phase contrast images are shown (magnification, x200). $\mathrm{P}<0.05$ pre-hypoxia vs. post-hypoxia groups. CA, compound anisodine; OD, optical density; PRCs, retinal progenitor cells; BNSCs, brain neural stem cells; C4, pre-treated with $1.010 \mathrm{~g} / 1 \mathrm{CA}$. 


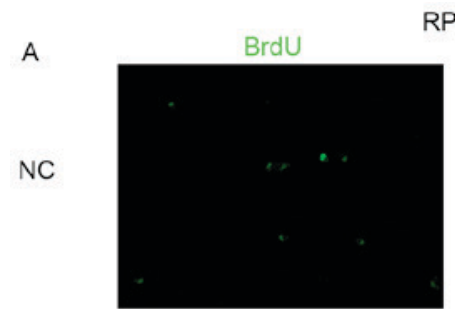

RPCs
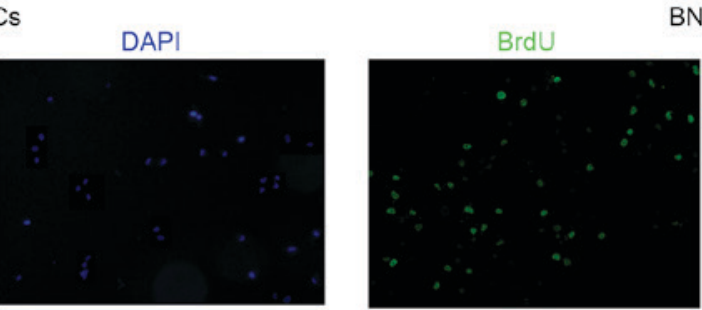

BNSCs
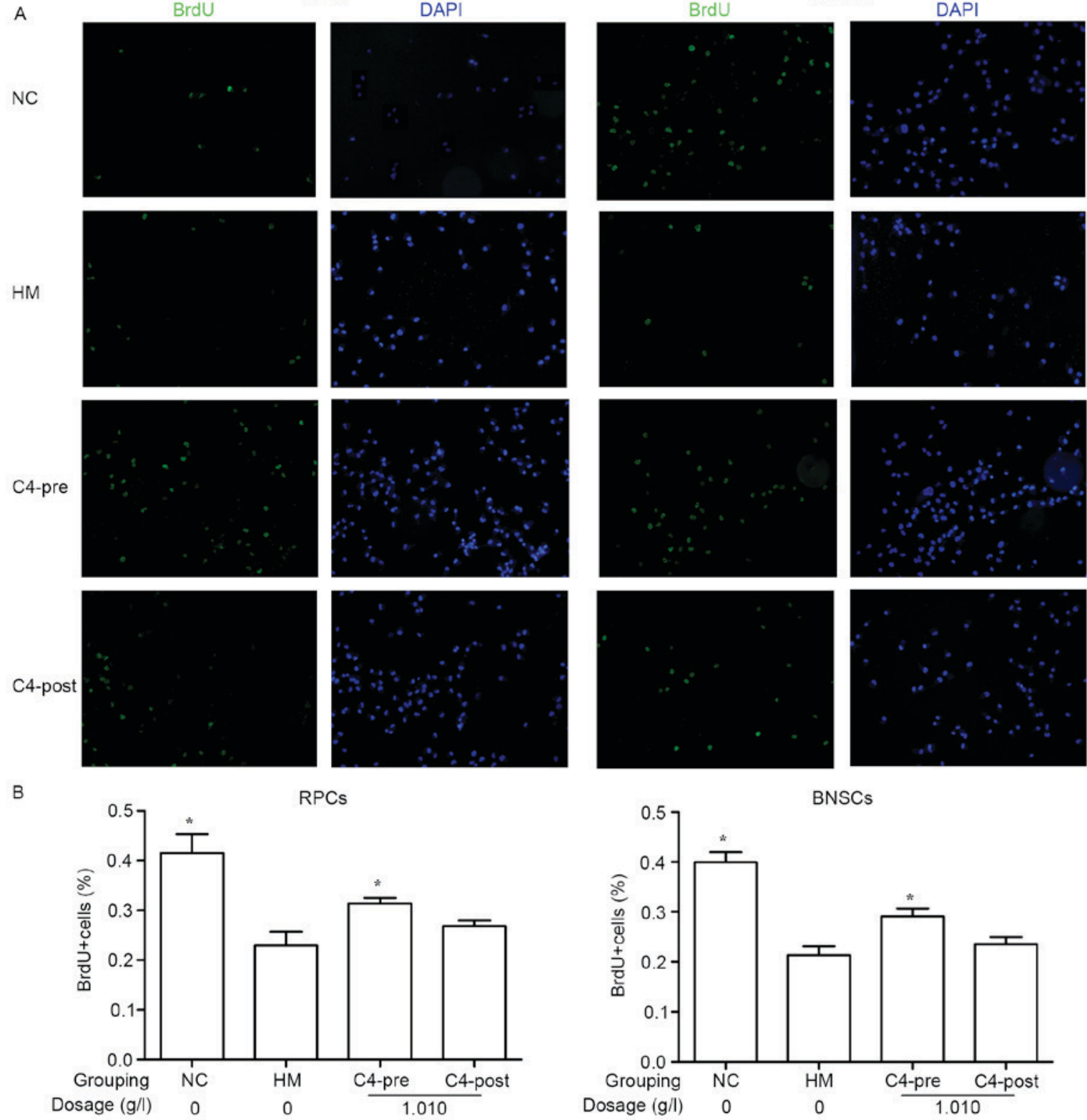

Figure 3. Effects of $1.010 \mathrm{~g} / 1 \mathrm{CA}$ on hypoxia-induced RPC and BNSC proliferation. (A) Wide-field microscopy analysis of BrdU incorporation in RPCs and BNSCs treated with $1.010 \mathrm{~g} / 1$ prior to and after hypoxia. BrdU immunocytochemistry (green) was perfomed $4 \mathrm{~h}$ after addition of BrdU. (B) Quantification of BrdU incorporation as representatively shown in A. Cells displaying a clear nuclear BrdU signal were counted as positive (magnification, $\mathrm{x} 400$ ). ${ }^{*} \mathrm{P}<0.05$ compared with HM group. BrdU, bromodeoxyuridine; PRCs, retinal progenitor cells; BNSCs, brain neural stem cells; HM, hypoxia model; NC, negative control; pre, pre-hypoxia; post, post-hypoxia; C4, pre-treated with $1.010 \mathrm{~g} / \mathrm{l} \mathrm{CA}$; CA, compound anisodine.

treatment was performed prior to or after hypoxia, the cell viability of RPCs was better than that of BNSCs in the $\mathrm{C} 2, \mathrm{C} 3$ and $\mathrm{C} 4$ concentration groups $(\mathrm{P}<0.05$; Fig. 2). These results suggested that 0.126-1.010 g/l CA may protect RPCs and BNSCs against hypoxia-induced apoptosis, and treatment prior to hypoxia showed superiority, with RPCs being more sensitive to $\mathrm{CA}$, as regardless of whether CA treatment was performed prior to or following hypoxia, the cell viability of RPCs was higher compared with the BNSCs in the C2, C3 and $\mathrm{C} 4$ concentration groups.

CA attenuates hypoxia-induced decreases in RPC and BNSC proliferation. BrdU incorporation of the cells was assessed after $4 \mathrm{~h}$ of incubation. Immunocytochemical staining indicated that after culture in a hypoxia incubator $(<1 \%$ oxygen) for $4 \mathrm{~h}$, the BrdU incorporation in RPCs $(22.94 \pm 2.77 \%)$ and BNSCs $(21.34 \pm 1.79 \%)$ was significantly decreased compared with that in the normal control groups $(41.58 \pm 3.71$ and $39.92 \pm 2.05 \%$, respectively). Treatment with $1.010 \mathrm{~g} / \mathrm{l} \mathrm{CA}$ for $4 \mathrm{~h}$ prior to hypoxia significantly improved the BrdU incorporation in RPCs $(31.41 \pm 1.08 \%)$ and BNSCs $(29.12 \pm 1.62 \%)$ compared with that in the HM group $(\mathrm{P}<0.05$; Fig. 3$)$.

CA attenuates hypoxia-induced cell cycle inhibition in RPCs and BNSCs. The influence of $1.010 \mathrm{~g} / \mathrm{l} \mathrm{CA}$ on the cell cycle of hypoxia-induced RPCs and BNSCs was investigated using FCM. The results demonstrated that after culture in a hypoxia incubator ( $<1 \%$ oxygen) for $4 \mathrm{~h}$, the $(\mathrm{S}+\mathrm{G} 2) \%$ of RPCs and BNSCs was significantly decreased as compared with that in the normal control group $(7.29 \pm 0.65$ vs. $15.11 \pm 0.45 \%$ for 

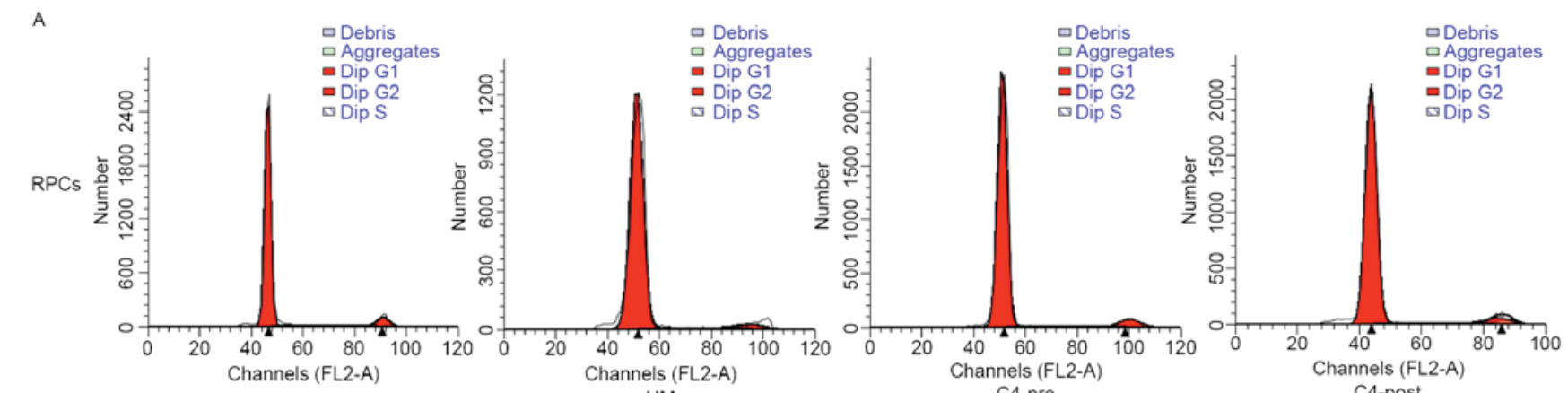

$\mathrm{NC}$

$\mathrm{HM}$

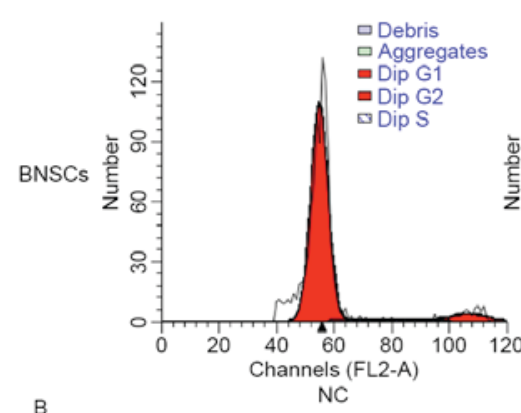

C4-pre

C4-post

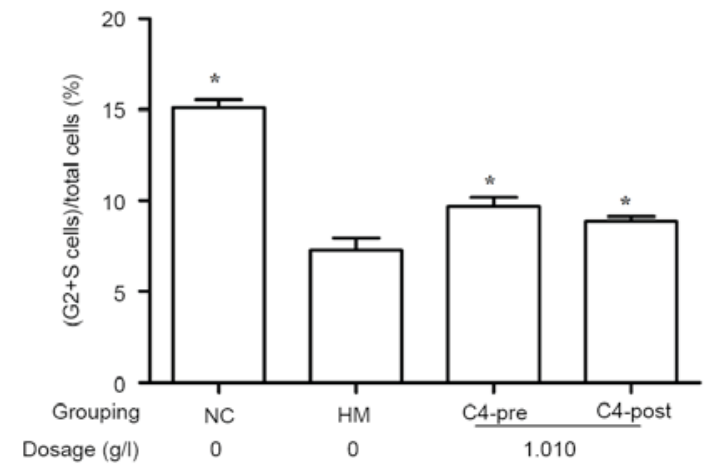

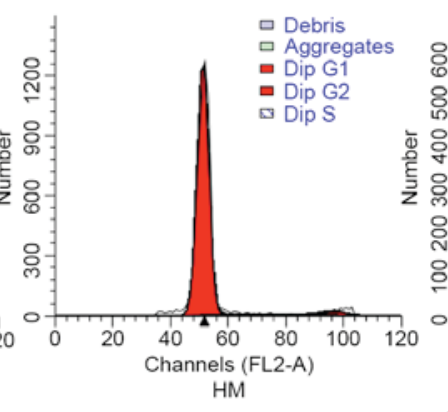

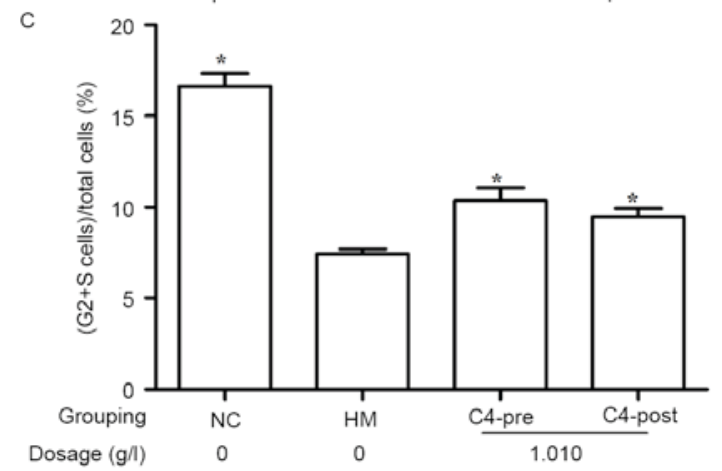

Figure 4. Effects of $1.010 \mathrm{~g} / \mathrm{l} \mathrm{CA}$ on the cell cycle of hypoxia-induced RPCs and BNSCs. (A) The representative fluorescence-assisted cell sorting plots displayed differences in cell cycle phases of RPCs and BNSCs. (B) Cell cycle analysis demonstrated that after treatment with $1.010 \mathrm{~g} / 1 \mathrm{CA}$, the proliferative index (the percentage of cells in S- and G2-phases) was significantly elevated when compared to that in the HM group. Data were derived from three independent experiments and are expressed as the mean \pm standard deviation. " $\mathrm{P}<0.05$ vs. HM group. PRCs, retinal progenitor cells; BNSCs, brain neural stem cells; $\mathrm{HM}$, hypoxia model; NC, negative control; pre, pre-hypoxia; post, post-hypoxia; C4, pre-treated with $1.010 \mathrm{~g} / 1 \mathrm{CA}$; CA, compound anisodine.

RPCs and $7.45 \pm 0.25$ vs. $16.63 \pm 0.71 \%$ for BNSCs; $\mathrm{P}<0.05)$. Furthermore, treatment with $1.010 \mathrm{~g} / \mathrm{l} \mathrm{CA}$ for $4 \mathrm{~h}$ prior to or after hypoxia significantly increased the $(\mathrm{S}+\mathrm{G} 2) \%$ of RPCs (9.68 $\pm 0.51 \%$ in the C4 Pre-hypoxia group; $8.88 \pm 0.24 \%$ in the C4 Post-hypoxia group) and BNSCs $(10.37 \pm 0.69 \%$ in the C4 Pre-hypoxia group; $9.48 \pm 0.47 \%$ in the $\mathrm{C} 4$ Post-hypoxia group) compared with that in the HM group ( $\mathrm{P}<0.05$; Fig. 4).

CA inhibits hypoxia-induced $\mathrm{Ca}^{2+}$ overload in RPCs and BNSCs. $\left[\mathrm{Ca}^{2+}\right]_{\mathrm{i}}$ was measured using Fluo 4-AM with detection of intracellular calcium fluorescence intensity by LSCM. The results showed that the $\left[\mathrm{Ca}^{2+}\right]_{\mathrm{i}}$ in hypoxia-cultured RPCs and BNSCs was markedly increased, and that $0.126-1.010 \mathrm{~g} / 1 \mathrm{CA}$ elicited concentration-dependent decreases in $\left[\mathrm{Ca}^{2+}\right]_{i}$. For the $\mathrm{C} 2$ and $\mathrm{C} 4$ concentration groups of RPCs and the $\mathrm{C} 3$ and $\mathrm{C} 4$ concentration groups of BNSCs, the $\left[\mathrm{Ca}^{2+}\right]_{i}$ in the pre-hypoxia group was significantly decreased as compared with that in the post-hypoxia group $(\mathrm{P}<0.05)$. Regardless of whether $\mathrm{CA}$ treatment was performed prior to or after hypoxia, the decreasing effect of CA on $\left[\mathrm{Ca}^{2+}\right]_{\mathrm{i}}$ in RPCs was greater than that in BNSCs in the $\mathrm{C} 1$ and $\mathrm{C} 2$ concentration groups $(\mathrm{P}<0.05$; Fig. 5$)$.
CA attenuates hypoxia-induced changes in Cyclin D1, HIF-1 $\alpha, V E G F$ and p-ERK in RPCs and BNSCs. Western blot analysis demonstrated that HIF- $1 \alpha$ and VEGF protein levels in the hypoxia-induced RPCs and BNSCs were upregulated, whereas Cyclin D1 expression levels and p-ERK were downregulated $(\mathrm{P}<0.05)$. After treatment with $1.010 \mathrm{~g} / \mathrm{l} \mathrm{CA}$, the expression of HIF-1 $\alpha$ and VEGF was downregulated, whereas Cyclin D1 and p-ERK were upregulated in both cell types compared with those in the HM group $(\mathrm{P}<0.05$; Figs. 6 and 7). However, t-ERK was not significantly affected by any of the treatments.

\section{Discussion}

A variety of ocular diseases, including diabetic retinopathy, retinopathy of prematurity, glaucoma and age-associated macular degeneration, which are often caused by ischemia, hypoxia and ischemic reperfusion of retinal lesions, lead to a hypoxic microenvironment in damaged regions of the retina, accompanied with changes of a series of cytokines and chemical substances. When transplanted, it is inevitable that the capabilities of 


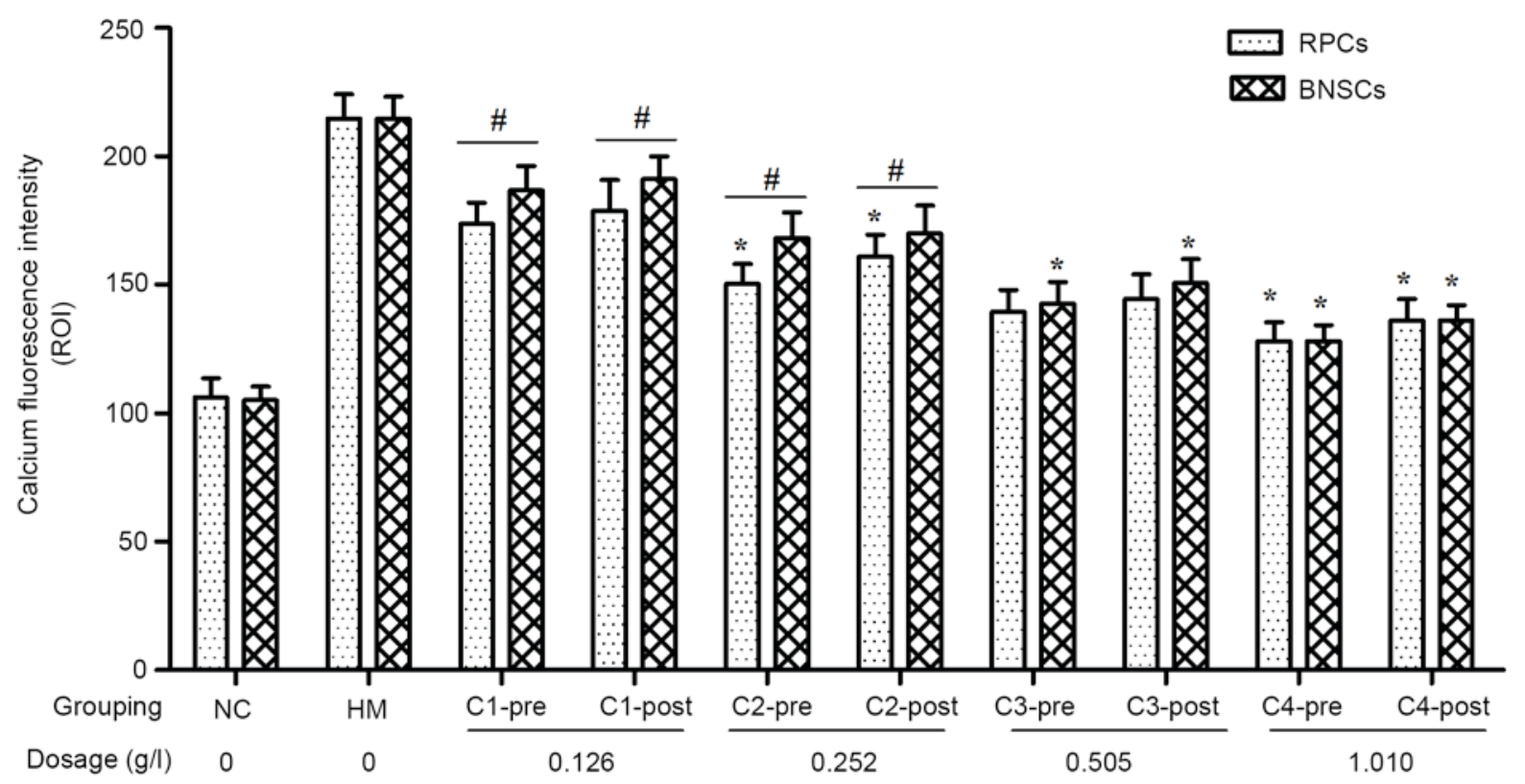

Figure 5. Effect of various concentrations of CA on intracellular calcium ion fluorescence intensity of RPCs and BNSCs before and after hypoxia. "P<0.05 pre-hypoxia vs. post-hypoxia groups; ${ }^{\#} \mathrm{P}<0.05 \mathrm{RPCs}$ vs. BNSCs. PRCs, retinal progenitor cells; BNSCs, brain neural stem cells; HM, hypoxia model; NC, negative control; pre, pre-hypoxia; post, post-hypoxia; C1-4, pre-treated with $0.126,0.252,0.505$ or $1.010 \mathrm{~g} / 1 \mathrm{CA}$, respectively; CA, compound anisodine.

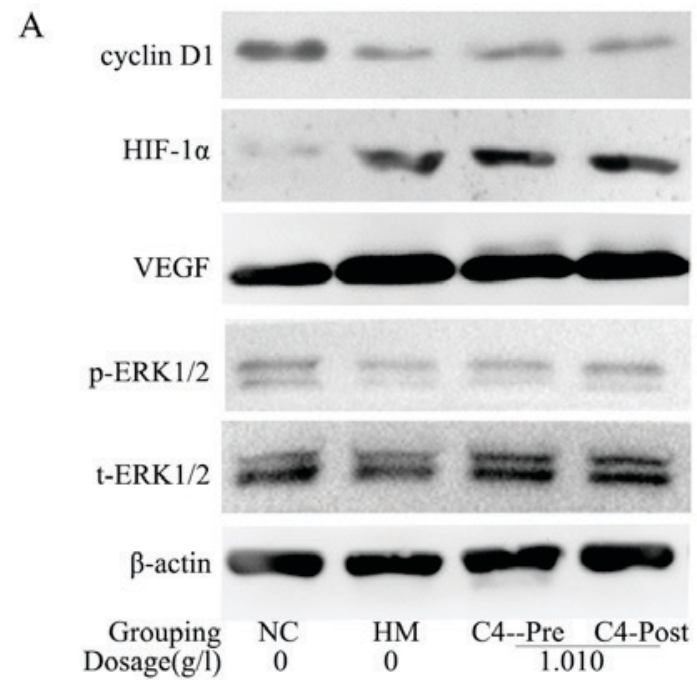

Figure 6. CA affects the expression of Cyclin D1, HIF-1 $\alpha$, VEGF, p-ERK and ERK in hypoxia-induced RPCs. (A) Representative western blots showing the protein expression levels of Cyclin D1, HIF-1 $\alpha$, VEGF, p-ERK and ERK in hypoxia-induced RPCs; (B) Quantified results of the levels of Cyclin D1, HIF-1 $\alpha$, VEGF, p-ERK and ERK protein in the NC, HM, C4-Pre and C4-Post groups of RPCs. Data were derived from three independent experiments and are expressed as the mean \pm standard deviation. ${ }^{*} \mathrm{P}<0.05$ vs. HM group; ${ }^{*} \mathrm{P}<0.05 \mathrm{C} 4$-pre vs. C4-post group. HIF, hypoxia-inducible factor; p-ERK, phosphorylated extracellular signal-regulated kinase; t, total; VEGF, vascular endothelial growth factor; PRCs, retinal progenitor cells; HM, hypoxia model; NC, negative control; pre, pre-hypoxia; post, post-hypoxia; C4, pre-treated with $1.010 \mathrm{~g} / 1 \mathrm{CA}$; CA, compound anisodine.

stem cells to proliferate, differentiate, migrate and integrate are affected by these substances (16). Numerous studies have confirmed that severe hypoxia caused the increase of intracellular reactive oxygen species (ROS), mitochondrial damage, and calcium overload, eventually leading to cell apoptosis. However, it has become obvious that hypoxia has a fundamental role in the maintenance of the stem cell niche. Emerging evidence suggested that low oxygen benefits the self-renewal of human embryonic, mesenchymal, hematopoietic, retinal and brain neural stem cells, as well as improving the efficiency of genetic reprogramming to induced pluripotency (17).
The oxygen concentration in mammalian tissues varies from $0.5 \%$ (retina) to $19 \%$ (upper airway epithelia), while it is maintained at $20 \%$ during routine cell culture. In adult retina, the oxygen concentration varies from $0.5 \%$ (inner nuclear layer) to $7 \%$ (outer segments) (18-20). Over the last decade it has become increasingly evident that the physiological condition of mild hypoxia $\left(2.5-5.0 \% \mathrm{O}_{2}\right)$ typical of neural tissues promotes the self-renewal of NSC (21); it also favors the success of engraftment when in vitro-expanded NSCs are transplanted into the brain of experimental animals. On the other hand, the cell death of NSCs increases under lower oxygen conditions 
A

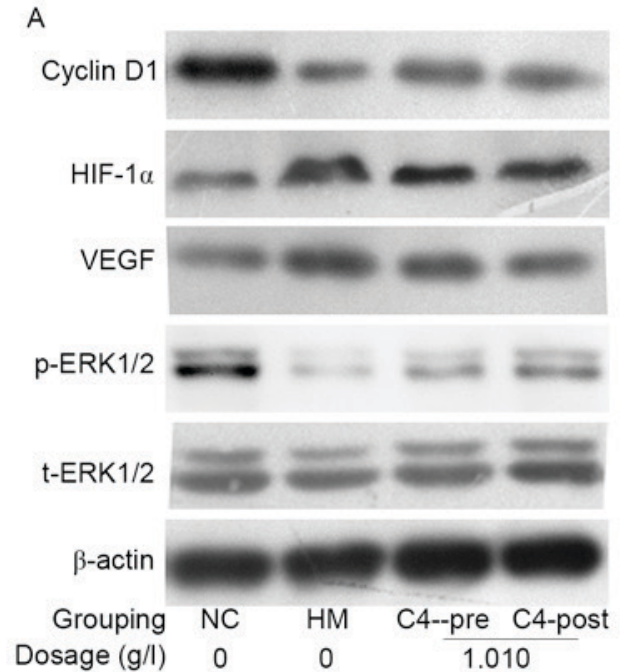

B

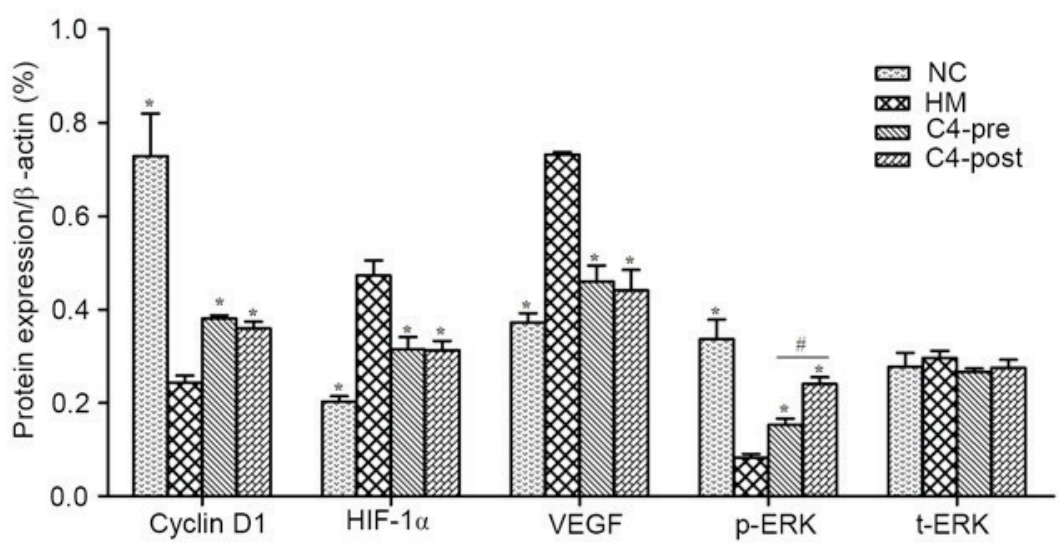

Figure 7. CA affects the expression of Cyclin D1, HIF-1 $\alpha$, VEGF, p-ERK and ERK in hypoxia-induced BNSCs. (A) Representative western blots showing the protein expression levels of Cyclin D1, HIF-1 $\alpha$, VEGF, p-ERK and ERK in hypoxia-induced BNSCs. (B) Quantified results of the levels of Cyclin D1, HIF-1 $\alpha$, VEGF, p-ERK and ERK protein in the NC, HM, C4-Pre and C4-Post groups of BNSCs. Data were derived from three independent experiments and are expressed as the mean \pm standard deviation. ${ }^{*} \mathrm{P}<0.05$ vs. hypoxia model group; ${ }^{*} \mathrm{P}<0.05 \mathrm{C} 4$-Pre vs. C4-Post groups. HIF, hypoxia-inducible factor; $\mathrm{p}$-ERK, phosphorylated extracellular signal-regulated kinase; t, total; VEGF, vascular endothelial growth factor; BNSCs, brain neural stem cells; HM, hypoxia model; $\mathrm{NC}$, negative control; pre, pre-hypoxia; post, post-hypoxia; C4, pre-treated with $1.010 \mathrm{~g} / \mathrm{l} \mathrm{CA}$; CA, compound anisodine.

$\left(<1 \% \mathrm{O}_{2}\right)$ and peaks at anoxia $\left(0 \% \mathrm{O}_{2}\right)$. In the present study, an oxygen concentration of $<1 \%$ was selected for the experiments and the aim of the study was to investigate the neuroprotective effects of CA on the proliferation and calcium overload of hypoxia-induced RPCs and BNSCs. In the present study, observation showed that after culture in a hypoxia incubator ( $<1 \%$ oxygen) for $4 \mathrm{~h}$, the neurospheres clumped together into large and irregular clusters, which eventually became dark in the center; at the same time, increasing amounts of floating cell debris were visible in the medium, indicating that an increased number of cells were dying. Furthermore, the results showed a reduction in cell proliferation and viability as well as an increase in the $\left[\mathrm{Ca}^{2+}\right]_{\mathrm{i}}$, in the hypoxia-induced RPCs and BNSCs.

In a previous clinical study (12-15), CA showed a favorable neuroprotective efficacy in various types of chemic optic neuropathy and choroidoretinopathy, particularly in the protection of the optic nerve in glaucoma and optic nerve contusion, which indicated that CA may have a protective effect on neurons and neural stem cells. Studies have shown that CA is capable to relieve angiospasm and increasing ocular blood flow, and to have anti-oxidant effects $(22,23)$. Moreover, Liu et al (24) suggested that oral administration of CA protects the function of retinal ganglion cell bodies and axons by increasing their survival rates in a mouse model with high intraocular pressure (24). In the present study, 0.126-1.010 g/l CA improved the viability and proliferation of hypoxia-induced RPCs and BNSCs, and protected the two cell types against hypoxia-induced calcium overload.

ERK1/2/HIF-1 $\alpha / \mathrm{VEGF}$ signaling is a critical pathway of physiological responses to acute and chronic hypoxia. HIF-1 $\alpha$ is a heterodimeric transcription factor, composed of two subunits, the HIF-1 $\alpha$ (or its analogs HIF-2 $\alpha$ and $-3 \alpha$ ) and HIF-1 $\beta$ subunits. HIF- $1 \alpha$ is an oxygen-sensitive subunit and its expression is induced under hypoxic conditions (25). In the present study, after culture in a hypoxia incubator ( $<1 \%$ oxygen) for $4 \mathrm{~h}$, the protein levels of HIF-1 $\alpha$ and VEGF were increased in RPCs and BNSCs. The ERK1/2 pathway is involved in hypoxia-induced HIF-1 $\alpha$ protein expression. Cyclin D1, as the most significant positive regulatory factor of the cell cycle, has a key role in $\mathrm{G} 1$ phase regulation and $\mathrm{G} 1 / \mathrm{S}$ phase transition. In the HM groups, the $(\mathrm{S}+\mathrm{G} 2) \%$ was decreased, indicating that cell mitosis was suppressed, and Cyclin D1 expression levels were downregulated. However, after treatment with $1.010 \mathrm{~g} / 1$ CA, the expression of HIF-1 $\alpha$ and VEGF was downregulated, whereas p-ERK and Cyclin D1 expression levels were upregulated in both cell types compared with those in the HM groups.

In conclusion, the results suggested that $0.126-1.010 \mathrm{~g} / \mathrm{l}$ $\mathrm{CA}$ attenuated the hypoxia-induced inhibition of proliferation of RPCs and BNSCs and protected against hypoxia-induced calcium overload by altering the protein expression levels of Cyclin D1 as well as hypoxia-associated proteins: HIF-1 $\alpha$, VEGF and p-ERK. However, assessing the influence of CA on the hypoxia tolerance of RPCs and BNSCs based on the experimental results in vitro may not be representative of the true effect of the complex microenvironment during retinal stem cell transplantation. Future experiments will involve detailed analysis of cellular and molecular events contributing to long-term effects of CA, including the association between HIF-1 $\alpha$ and intracellular calcium ions.

While studies in the retinal stem cell field have led to the identification of various cell sources and methods to enhance transplant cell migration and integration, a large amount of research is required for the implementation of cell-based therapies for treating human retinal disease.

\section{References}

1. Garcia JM, Mendonça L, Brant R, Abud M, Regatieri C and Diniz B: Stem cell therapy for retinal diseases. World J Stem Cells 7: 160-164, 2015.

2. Balmer J, Stanzel BV and Fischer MD: Stem cell therapy for retinal diseases. Ophthalmologe 112: 728-737, 2015 (In German). 
3. Ng TK, Fortino VR, Pelaez D and Cheung HS: Progress of mesenchymal stem cell therapy for neural and retinal diseases. World J Stem Cells 6: 111-119, 2014.

4. Pellegrini G, De Luca M and Arsenijevic Y: Towards therapeutic application of ocular stem cells. Semin Cell Dev Biol 18: 805-818, 2007.

5. Yu D and Silva GA: Stem cell sources and therapeutic approaches for central nervous system and neural retinal disorders. Neurosurg Focus 24: E11, 2008.

6. Dunn-Thomas TE, Dobbs DL, Sakaguchi DS, Young MJ, Honovar VG and Greenlee MH: Proteomic differentiation between murine retinal and brain-derived progenitor cells. Stem Cells Dev 17: 119-131, 2008.

7. Qu Z, Guan Y, Cui L, Song J, Gu J, Zhao H, Xu L, Lu L, Jin Y and Xu GT: Transplantation of rat embryonic stem cell-derived retinal progenitor cells preserves the retinal structure and function in rat retinal degeneration. Stem Cell Res Ther 6: 219, 2015.

8. Chacko DM, Das AV, Zhao X, James J, Bhattacharya S and Ahmad I: Transplantation of ocular stem cells: The role of injury in incorporation and differentiation of grafted cells in the retina. Vision Res 43: 937-946, 2003.

9. Francis PJ, Wang S, Zhang Y, Brown A, Hwang T, McFarland TJ, Jeffrey BG, Lu B, Wright L, Appukuttan B, et al: Subretinal transplantation of forebrain progenitor cells in nonhuman primates: Survival and intact retinal function. Invest Ophthalmol Vis Sci 50: 3425-3431, 2009.

10. Guo Y, Saloupis P, Shaw SJ and Rickman DW: Engraftment of adult neural progenitor cells transplanted to rat retina injured by transient ischemia. Invest Ophthalmol Vis Sci 44: 3194-3201, 2003.

11. Nishida A, Takahashi M, Tanihara H, Nakano I, Takahashi JB, Mizoguchi A, Ide C and Honda Y: Incorporation and differentiation of hippocampus-derived neural stem cells transplanted in injured adult rat retina. Invest Ophthalmol Vis Sci 41: 4268-4274, 2000.

12. Chen YH and Wu LQ: Therapeutic effect of compound anisodine for primary open angle glaucoma. Zhejiang Da Xue Xue Bao Yi Xue Ban 40: 659-662, 2011 (In Chinese).

13. Wang W, Huang Y, Zhang J, Jiang J and Huang J: Efficacy of cytidine-5'-diphosp-bocholine combined with compound anisodine in the treatment of early optic nerve contusion. Eye science 27: 37-40, 2012
14. Wang Z: Observation on clinical application of compound Anisodine for patients with optic atrophy disease. Huli Yanjiu 24: $1657,2010$.

15. Yi CM, Yu HY, Zhang L, Mfng W, Peng HY, Liu LB and Yang CY: Clinical observation of compound anisodine treating for ischemic ophthalmopathy. Inner Mongolia Med J 1: 011, 31-34, 2008.

16. Kelley MW, Turner JK and Reh TA: Regulation of proliferation and photoreceptor differentiation in fetal human retinal cell cultures. Invest Ophthalmol Vis Sci 36: 1280-1289, 1995.

17. Clarke $L$ and van der Kooy D: Low oxygen enhances primitive and definitive neural stem cell colony formation by inhibiting distinct cell death pathways. Stem Cells 27: 1879-1886, 2009.

18. Baranov PY, Tucker BA and Young MJ: Low-oxygen culture conditions extend the multipotent properties of human retinal progenitor cells. Tissue Eng Part A 20: 1465-1475, 2014.

19. Yu DY and Cringle SJ: Oxygen distribution in the mouse retina. Invest Ophthalmol Vis Sci 47: 1109-1112, 2006.

20. Cringle SJ and Yu DY: Oxygen supply and consumption in the retina: Implications for studies of retinopathy of prematurity. Doc Ophthalmol 120: 99-109, 2010.

21. Guo Z, Shi F, Zhang L, Zhang H, Yang J, Li B, Jia J, Wang X and Wang X: Critical role of L-type voltage-dependent $\mathrm{Ca} 2+$ channels in neural progenitor cell proliferation induced by hypoxia. Neurosci Lett 478: 156-160, 2010.

22. Song C, Shen W and He Q: An experimental study on compound anisodine III for softening scar of mouse skin after burn. Zhonghua Yan Ke Za Zhi 32: 176-178, 1996 (In Chinese).

23. Zhu Y, Song $\mathrm{C}$ and Wang S: The changes of hemodynamics in ocular trauma and treatment with compound anisodine. Zhonghua Yan Ke Za Zhi 32: 110-113, 1996 (In Chinese).

24. Liu WD, Chen LL, Shen CY and Jiang LB: Neuroprotective effect of compound anisodine in a mouse model with chronic ocular hypertension. Chin Med J (Engl) 128: 2652-2657, 2015.

25. Ejtehadifar M, Shamsasenjan K, Movassaghpour A, Akbarzadehlaleh P, Dehdilani N, Abbasi P, Molaeipour Z and Saleh M: The effect of hypoxia on mesenchymal stem cell biology. Adv Pharm Bull 5: 141-149, 2015. 